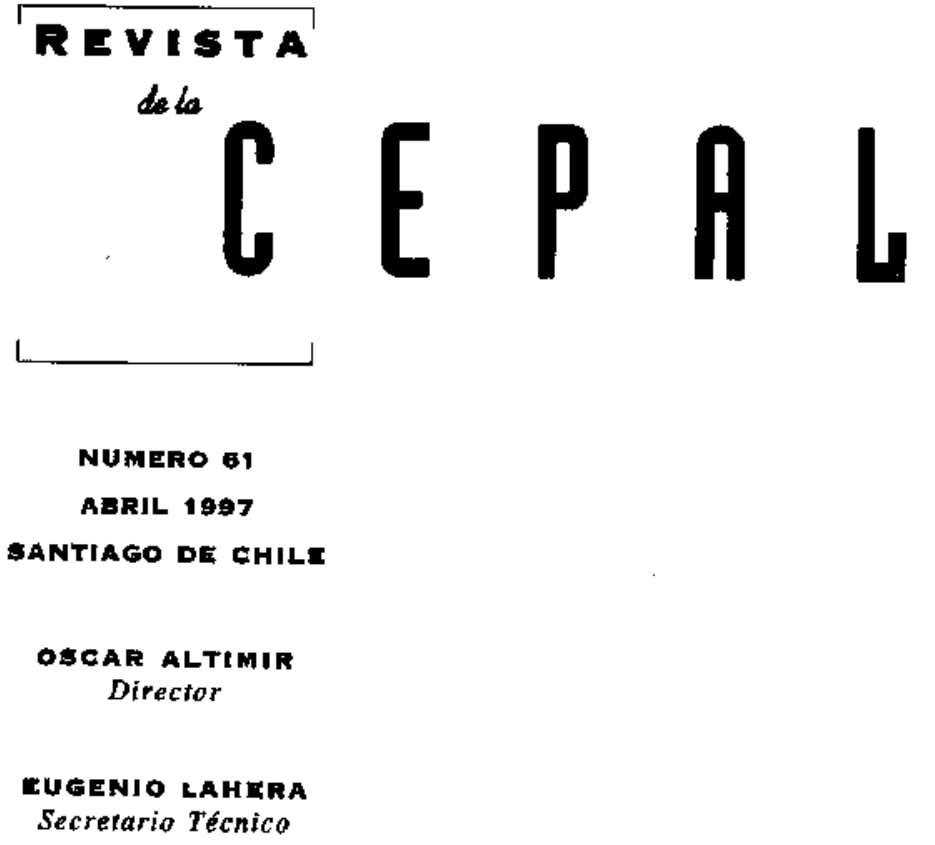

Secretario Técnico

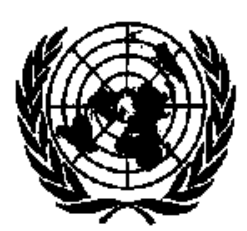

NACIONES UNIOAS 
Tres formas de coordinación social

Norbert Lechner

Brechas sociales en Colombia

Juan Luis Londoño de la Cuesta

Los Estados Unidos al rescate: la asistencia flnanciera a México en 1982 y 1995

Nora Lustig

El réglmen de convertibllidad y el sistema bancario en la Argentina Alfredo F. Calcagno

Exportaciones de manufacturas: desafíos para las pequeñas economías de América Latina

Rudolf M. Buitelaar, Pitou van Dijck

¿Por qué las inversiones en el transporte público no reducen la congestión de tránsito urbano?

lan Thomson

Nolas sobre la mediclón de la pobreza según el método del ingreso

Juan Carlos Feres

La politica fiscal y el ciclo económico en Chile

Carlos Budnevich, Guillermo Le Fort

Evaluación de la política de bienes de capital en la Argentina

Pablo Sirlin

Reestructuraclón de los grupos industriales brasileños entre 1980 y 1993

Ricardo $M$. Ruiz

Reestructuración productiva y cambio territorial: un segundo eje de Industrlallzación en el norte de México

Tito Alegría, Jorge Carrillo, Jorge Alonso Estrada

Publicaciones recientes de la CEPAL 


\section{La política fiscal y el ciclo económico en Chile}

\section{Carlos Budnevich* Guillermo Le Fort"*}

*Gerente de Análisis

Financiero,

Banco Central de Chile

**Gerente de División

Internacional,

Banco Central de Chile
En este artículo se estudia el efecto de la estabilización del gasto fiscal y del uso anticíclico de los impuestos como variables de estabilización de la economfa chilena, a través de la calibración de un modelo macroeconónico básico ajustado a la realidad de dicha economía; los resultados demuestran que alrededor de un $25 \%$ de la variabilidad del crecimiento economico podría ser eliminada evitando los impulsos cíclicos fiscales a traves de un crecimiento constante de la inversión y el consumo público, así como también a través de tributos anticíclicos. Se propone, por un lado, establecer un sistema de estabilización del crecimiento del gasto fiscal mediante un sistema de reglas y grados de flexibilidad sujetos a cláusulas específicas; un elemento de importancia en la implementación de las políticas estabilizadoras es un fondo de estabilización de la recaudación tributaria en el que se contabilizarían y acumularían las respuestas contracíclicas del fisco y se independizaria el gasto fiscal de la recaudación en un punto particular del ciclo económico. De otro lado, se estudia tanto la factibilidad y eficacia de flexibilizar la fijación del impuesto al valor agregado (IVA), como los problemas de inconsistencia temporal que de ello derivarfan. Por esta razón se consideran los costos y beneficios de complementar la flexibilidad del IVA con mayores grados de flexibilidad del impuesto a la renta o de los incentivos tributarios a la inversión. Se concluye que en manos del fisco hay una serie de instrumentos que se podrían usar para llevar adelante una política anticíclica, como es la posibilidad de establecer reglas sobre crecimiento del gasto, fondos de estabilización y flexibilidad de los impuestos. 
I

\section{Introducción}

En los últimos 11 años la economfa chilena ha exhibido una expansión sostenida. La tasa de crecimiento medio del PIB en esos años ha superado el $6.5 \%$, cifra muy superior al promedio historico chileno. Sin em. bargo este crecimiento, pese a ser sostenido y a tasa elevada, ha presentado importantes variaciones cíclicas, con tasas de expansión que han variado entre $3.3 \%$ en 1990 y $11 \%$ en 1992 (gráfico 1).

Se puede atribuir este comportamiento cíclico a diversos orígenes, entre ellos los frecuentes shocks a los que ha estado sometida la economía chilena en este período y en casi toda su historia independiente. ${ }^{1} \mathrm{En}$ tre estos shocks se hallan tanto los de origen externo, por cambios en las condiciones de la economía internacional, como los de origen interno, incluidos los efectos de modificaciones en las políticas macroeconómicas.

Existe un relativo consenso en que estos vaivenes cíclicos que afectan a la actividad económica real son costosos para la economía como un todo, comparados con una situación hipotética de crecimiento estable a la tasa media. La variabilidad del crecimiento introduce un mayor riesgo en la economía que puede influir negativamente en la inversión y en las posibilidades de crecimiento futuras. Además, algunos de los efectos de una tasa de crecimiento transitoriamente baja no son completamente compensados en los períodos de crecimiento alto. En particular, la reducción de la actividad y el empleo durante el período de bajo crecimiento puede afectar de manera importante la acumulación de capital humano de los sectores más pobres.

Dado que la inestabilidad cíclica aparece como indeseable, cabe preguntarse si la política pública cuenta con elementos para suavizar tales variaciones. Aquí analizaremos la posibilidad de que la política fiscal se

$\square$ Una versión preliminar de este trabajo fue presentada en un seminario realizado en enero de 1996 en el Banco Central. Se agradecen los comentarios de José de Gregorio y Gonzalo Sanhueza y la valiosa colaboración de Oscar Landertetche M. y Sergio Godoy. El contenido de este trabajo refleja opiniones personales de sus alltores, las que en ningún caso comprometen a la institución a la que pertenecen,

Véase una interesante reseña de los shocks experimentados por la economfa nacional a lo largo de su historia, en Cortés, 1984.
GRATICO I

Chile: Tașa de crecimiento anual del producto interno bruto entre 1988 y 1993

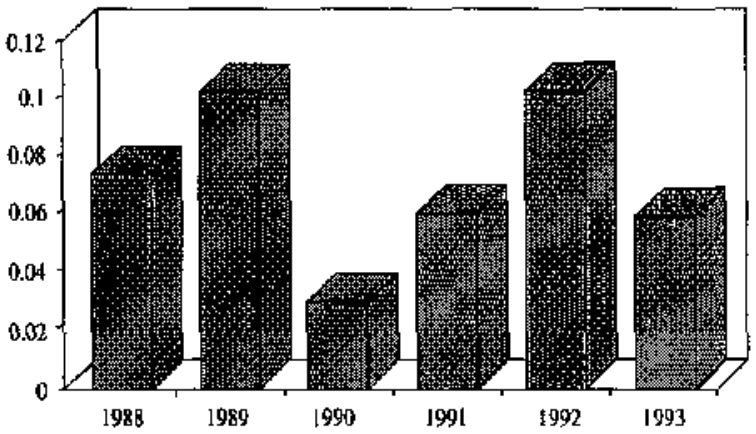

defina sobre la base de un esquema dirigido a la moderación del ciclo. Esto porque la política monetaria tiene como objetivo prioritario el control de la inflación. Nos preguntamos si la estabilización de la tasa de expansión del gasto público en torno a la tasa de crecimiento del producto potencial puede tener o no un efecto significativo sobre la variabilidad del crecimiento del PB. Cabe preguntarse también si una política tributaria más flexible y definida en términos contracíclicos puede ser eficaz en tal sentido. Para dar respuesta a estas interrogantes es necesario evaluar cuantitativamente los efectos que las políticas de gasto público y tributarias tienen sobre el crecimiento cíclico del producto.

Es interesante preguntarse asimismo hasta qué punto una política fiscal definida sobre la base de las condiciones de mediano y largo plazo modera el efecto cf́clico, en comparación con una política de gasto definida sobre la base de los ingresos anuales. Si el gasto depende del ingreso coyunturalmente disponible, y éste a su vez depende del ciclo económico, parece muy plausible que una redefinición de la política de gasto tenga efectos moderadores de la variabilidad cíclica.

En las secciones que siguen se evalúa el efecto que tendrían diversas políticas en el gasto público, así como el que tendría el manejo de los tributos indirectos sobre la estabilidad del crecimiento. Ante todo, se pasa revista a los principales hechos macroeconómicos que marcaron el período 1988-1993 (sección II). Lue- 
go se describe brevemente el modelo que se usó para realizar las simulaciones con las cuales se verifican los efectos de las distintas opciones de política fiscal sobre la estabilidad del crecimiento y se presentan los resultados principales de las distintas simulaciones (sección III), y finalmente, se resumen las principales conclusiones del estudio (sección IV).

Este trabajo no aborda muchos de los aspectos importantes relativos a la definición de la política fis. cal, entre ellos las medidas para optimizar la eficacia de los distintos programas de gasto, el tamaño del Estado, el nivel de gasto público y los objetivos que con Él se persiguen. Cabe destacar, eso sí, que la adopción de alguna regla respecto del ritmo de crecimiento del gasto público disminuye la incertidumbre acerca de esta importante variable macroeconómica. El temor de algunos sectores en relación a que el tamaño del sector público aumente y que el manejo del presupuesto origine inestabilidad económica e inflación - lo que tiene fundamentos en experiencias pasadas vividas en Chile y otros parses del mundo- resiente la inversión y frena el crecimiento.

Estas consideraciones apuntan a la conveniencia de que el gobierno disponga de herramientas pata enfrentar circunstancias inesperadas, como las que provienen del exterior. La más importante de esas circunstancias es un eventual ciclo recesivo, frente al cual la existencia de un límite preestablecido al crecimiento del gasto público, en conjunto con las actuales dificultades para modificar los impuestos en forma ágil, ataría de manos a la autoridad fiscal. Por otra parte, si hubiese un doble calentamiento de la economía, la existencia de un compromiso formal de no hacer crecer el gasto público más que el producto obstaculizaría la adopción de una política de gasto transitoriamente contractiva, por cuanto ella no podría ser compensada por una política de gasto expansiva en años posteriores.

\section{II}

\section{La economía chilena en 1988-1993}

Comparado con los años anteriores, el sexenio 19881993 tuvo resultados muy favorables. La tasa de crecimiento del PIB medio anual super6 el $7 \%$, tras haber sido menos de $1 \%$ en el período 1982-1987. Ese mayor crecimiento se reflejó en una menor tasa de desempleo: sólo $6 \%$ en promedio, comparado con $14 \%$ en el sexenio anterior. La economía chilena no sólo ha logrado recuperarse plenamente de la crisis de los años ochenta, lo que se refleja en que el nivel del piB de 1981 se alcanzó nuevamente en 1986, sino que ha alcanzado un crecimiento sostenido a tasas muy superiores a las históricas.

El elevado crecimiento medio se ha dado junto con un marcado comportamiento ciclico, to que ha obligado a realizar en el período dos ajustes importantes sobre la base de la política monetaria. La tasa de interés de los instrumentos del Banco Central tuvo dos aumentos importantes en el período, primero en 1990 y luego a mediados de 1992, los que respondieron a tasas de crecimiento del producto y del gasto consideradas excesivas para los objetivos en materia de inflación y cuentas externas. La eficacia de tales procesos de ajuste ha permitido que este período de vigoroso crecimiento económico haya sido acompañado de ta- sas de inflación bajas para los estándares nacionales, y además declinantes. La inflación media del período analizado fue de $17.5 \%$ frente a $22 \%$ del sexenio anterior, y la tasa anual bajó desde un máximo de $27.3 \%$ en 1990 a menos de $10 \%$ en 1994. Al respecto cabe preguntarse si el apoyo de la política fiscal a estos objetivos ayudaría a disminuir la amplitud del ciclo económico, haciendo más fácil reducir la inflación en Chile a tasas comparables con la de los países industriales.

Los años 1988 y 1989 fueron claramente expansivos. En esos años el PIB se elev 6 en $7.3 \%$ y $10.2 \%$, respectivamente. La inflación, que en 1988 alcanzaba a $12.7 \%$, en 1989 aument6 a $21.4 \%$. La situación externa fue bastante favorable, con superávit en la balanza comercial de 2218.6 millones de dólares $(9.2 \%$ del PIB nominal) y 1578.1 millones $(5.6 \%$ del PIB nominal), respectivamente. El déficit en cuenta corriente fue de 167.4 millones de dólares ( $0.7 \%$ del PIB nominal) en 1988 y de 705 millones ( $2.5 \%$ del PrB nominal) en 1989; ambos se pudieron financiar sin problemas debido a la importante entrada de capitales que hubo en ese bienio. El sector público, por su parte, tuvo un comportamiento moderado en 1988, al crecer la absor- 
ción pública real en $6.4 \%$, y uno contractivo en 1989 , al reducirse la absorción pública real en $1 \%$.

Ante la expansión mencionada, el Banco Central -entidad independiente- decidió, el 8 de enero de 1990 , elevar la tasa de interés de sus valores a un año plaze de $8 \%$ a $9.7 \%$, y no alzar la tasa de corto plazo coino se hace actualmente, provocando así un shock monetario para reducir el gasto y controlar la inflación. El diagnóstico de las autoridades era que en los dos años anteriores se había producido una expansión de la demanda agregada que no era sostenible en el largo plazo. Esta alza de la tasa de interés, junto con una mejor percepción del sistema financiero internacional sobre el riesgo del paŕs, provocó una fuerte entrada de capitales; éstos fueron absorbidos por el Banco Central, que en el año aumentó sus reservas en $82 \%$.

A pesar de la política de ajuste monetario descrita, la inflación mostró bastante renuencia a bajar ese mismo año. Sin embargo, es necesario reconocer que en 1990 algunas alzas importantes conspiraron contra la política antiinflacionaria. ${ }^{2}$ Sus efectos en una economía altamente indexada, como lo es la chilena, tienden a prolongarse en el tiempo. La política monetaria restrictiva fue bastante eficaz en cuanto a reducir el nivel de actividad, lo que se notó en una baja de la tasa de crecimiento del PIB de $10.2 \%$ en 1989 a $3 \%$ en 1990 , debida sobre todo a una importante desaceleración del gasto interno, especialmente de consumo, tanto del gobiemo como privado. La situación externa, por su parte, fue bastante favorable, con una balanza comercial muy positiva del orden de 1273.1 millones de dólares (4.2\% del PIB nominal) y un déficit en cuenta corriente de 648.0 millones ( $1.9 \%$ del PIB nominal) perfectamente financiable gracias a la gran entrada de capitales, especialmente de corto plazo, cuyas causas explicamos más arriba. El sector público ayudó al ajuste de la economía en 1990 al caer la absorción pública en $5 \%$.

En 1991, el Banco Central fue disminuyendo gradualmente los tipos de interés de sus pagarés, en respuesta a la disminución observada de la actividad y a la reducción del proceso inflacionario. El PIB creció en $6.1 \%$, y la inflación por fin respondio a la política de ajuste, bajando a $18.7 \%$ desde el $27.3 \%$ del año anterior. ${ }^{3}$ En cuanto al sector externo, la balanza comer-

\footnotetext{
${ }^{2}$ Como la fuerte alza del precio del petróleo; el aumento del im puesto al valor agregado, y algunas presiones salariales como el alza (teal) del salario mínimo (casi $45 \%$ nominal en junio).

${ }^{3}$ Sin embargo, hay que reconocer la importancia que tuvo en esta reducción del ritmo inflacionario la baja de aranceles y la caida del precio del petróleo.
}

cial arrojó un saldo positivo del orden de los 1575.9 millones de dólares (4.64\% del plB nominal) y el défícit en cuenta corriente fue prácticamente nulo; pero continuó la fuerte entrada de capitales, reflejándose en un alza nominal de $24.2 \%$ en las reservas internacionales. Ante esta persistente entrada de recursos, a mediados de año el Banco Central decidió tomar medidas para restringir la entrada de divisas, especialmente las de más corto plazo. ${ }^{4} \mathrm{El}$ comportamiento del sector público fue mucho más expansivo que el año anterior: la absorción pública real se elev6 en $7.1 \%$.

Durante 1992 el PBB crecí en $10.3 \%$. El desempleo se redujo de $6.6 \%$ en 1991 a $4.9 \%$, un nivel históricamente muy bajo para la economfa chilena. La inflación descendió a $12.7 \% .^{5}$ La situación externa también fue muy favorable, con una batanza comercial de 749.2 millones de dólares (1.8\% del PIB nominal) y un déficit en la cuenta corriente de 743.0 millones (1.8\% del PIB nominal) que pudo financiarse sin problemas con la entrada de capitales que incluso permitió un aumento de las reservas internacionales. Por su lado, el sector público tuvo un comportamiento claramente expansivo, al aumentar la absorción pública real en $13.1 \%$.

La paulatina alza de la tasa de interés en 1992, y una demanda externa menos favorable hicieron que en 1993 la actividad económica redujera su ritmo de expansión; el PIB aumentó alrededor de un $6 \%$ y la tasa de desempleo descendio levemente, alcanzando el nivel más bajo en los últimos 20 años. La inflación, por su parte, tendió a bajar un poco respecto del año anterior. Debido al menor dinamismo del sector externo, lo que se tradujo en una carda de la relación de precios del intercambio de $8.5 \%$ aproximadamente, la balanza comercial tuvo por primera vez en más 10 años un saldo negativo, del orden de los 978.6 millones de dólares (2.2\% del pib nominal), y el déficit en la cuenta corriente llego a 2092.0 millones (4.8\% del PIB

\footnotetext{
4 Las medidas más importantes fueron la revaluaciôn de $2 \%$ mencionada, y el establecimiento de un encaje ao remunerado de $20 \%$ y de un af́o plazo para los créditos externos. Este permitió en alguna medida atenuar la entrada de capitales. Asimismo, la revaluación y la baja de aranceles correspondieron a un reconocimiento por parte de las autoridades económicas de que la fuerte entrada de divisas ocurrida en el último ậo y medio tenía un importante conponente estructural o permanente, y no transitorio como se habia diagnosticado ínicialmente.

${ }^{5}$ En este descenso influyó la apreciación nominal ocurtida durante el afio, que sólo alcanzo a $3.8 \%$, la tendencia sostenida a la baja del precio del petróleo y la gradual alza de la tasa de interés por parte del Banco Central, para desacelerar el gasto interno que parecia estar creciendo en demasía.
} 
nominal). Este déficit, a pesar de ser el más grande de la década, se pudo financiar sin ningún problema gracias a que la entrada de capitales continú, con lo cual incluso las reservas internacionales del Banco Central crecieron en poco más de $8 \%$, pasando de $9009 \mathrm{mi}$ - llones de dólares (21.8\% del PIB nominal) a $9759 \mathrm{mi}-$ llones (22.3\% del P(B nominal). Por su lado, el sector público mostró una actitud bastante más moderada que el año anterior, ayudando así en parte al ajuste con un crecimiento de la absorción pública real de $6.4 \%$.

\section{III}

\section{El modelo de simulación y sus resultados}

El modelo empleado en las simulaciones es un modelo macroeconómico simplificado, de corte keynesiano, ${ }^{6}$ que incluye sólo cuatro funciones de comportamiento: consumo privado real, excluido el gasto en consumo financiado directamente por transferencias del sector público; tributos reales, excluidos los ingresos públicos provenientes del cobre; importaciones de bienes y servicios en términos reales, y tipo de cambio real. Las demás variables de la demanda agregada, incluidas las transferencias del sector público al privado, la inversión privada, el consumo e inversión públicos y las exportaciones se consideran exógenas. Otras variables endógenas, tales como el PIB, se calculan sobre la base de identidades que incorporan tanto variables exógenas como de comportamiento.

El modelo es estrictamente real, es decir, ignora los efectos monetarios y financieros de las medidas empleadas, ya que no incluye el mercado monetario, ni la tasa de interés como argumento explicatorio de las funciones de comportamiento presentadas. Tampoco considera los efectos de riqueza que podría tener la política fiscal adoptada, ni un posible desplazamiento vía tasa de interés (crowding out) entre gasto de gobierno e inversión, ya que los efectos del mecanismo de la tasa de interés son ignorados por propósitos de simplificación y la inversión se considera exógena. Lo que este modelo sí incluye es el efecto directo del gasto público sobre el consumo privado (sin transferencias), porque al elevarse el gasto público crece el PIB, lo que a su vez aumenta el consumo privado. ${ }^{7}$ Este efecto es

${ }^{6}$ Modelo keynesiano a la manera de Blinder y Solow (1989), Christ (1967) y Frenkel y Razin (1987). La aplicación del modelo keynesiano en economía abierta con movilidad de capitales priva dos se examina en Mundell (1963), Fleming (1962) y Sachs y Larrain (1993).

${ }^{7}$ Este efecto es un posible resultado previsto por el modelo keynesiano más extremo, en el cual la oferta agregada es totalmente elástica y la inversión es insensible a la tasa de interés, por lo cual el efecto de un mayor gasto de gobiemo aumenta el producto compensado en parte por el incremento de los ingresos públicos que reduce el ingreso disponible.

Se define el consumo privado sin transferencias como el gasto en consumo privado excluidas las transferencias corrientes del sector público, las que son consideradas como ingreso de grupos que tienen una propensión marginal unitaria a consumir. El consumo privado sin transferencias es función del ingreso disponible privado -que se calcula como el ingreso nacional (PIB) más los ingresos netos de factores desde el resto del mundo, descontados los tributos--y del mismo consumo con un perfodo de rezago. ${ }^{8}$ Los tributos no vinculados al cobre, que incluyen todos los ingresos corrientes del fisco excepto los provenientes de la minería de ese metal, son función del PIB.

El tipo de cambio real (TRC) representa el precio relativo entre los bienes transables y los bienes no transables. Se le define como la razón entre los precios externos medidos en dolares - representados por el promedio ponderado de los índices de precios al por mayor de los principales socios comerciales medidos en dólares - y los precios internos medidos en dólares, representados por el índice de precios al consumidar dividido por el tipo de cambio nominal. En el modelo, la tasa de variación del tipo de cambio real es función inversa de la diferencia entre las tasas de cre-

demandado y ofrecido, lo que a igualdad de otras condiciones, eleva el consumo privado. Además, en este modelo, al crecer el pIB y la absorción aumentan las importaciones por dos causas. Prìmero, por el efecto directo del PIB, que se muestra más adelante en la ecuación (3); y segundo, porque al aumentar la absorción baja el tipo de cambio real: como el producto tendencial está dado, la relación entre éste y la absorcion disminuye, por lo que cae el tipo de cambio real - ver ecuación (4) más adelante- $y$ así suben las importaciones. Este aumento de las importaciones hace que el PIB disminuya, lo que también reduce el efecto del gasto público sobre el consumo privado (sin transferencias).

${ }^{8}$ El proceso autorregresivo del consumo puede explicarse tanto porque existen costos de ajustes en el consumo, como por la formación de expectativas respecto del ingreso futuro. 
cimiento del gasto agregado y del producto de tendencia. De esta forma, un crecimiento en el gasto que excede el crecimiento del producto de tendencia genera un exceso de demanda de bienes no transables y, por lo tanto, tiende a reducir el tipo de cambio real.

Por último, la demanda de importaciones es función del producto interno y del tipo de cambio real; reacciona ante cambios en la absorción a través del efecto que esta última tiene sobre el tipo de cambio real. Todas las ecuaciones fueron calibradas para simular el resultado efectivo. Esto se realizó agregando para cada variable endógena una serie representativa de shocks exógenos, que fue definida para que el modelo reprodujera fielmente los resultados observados en la realidad. Estas series fueron empleadas en las distintas simulaciones.

El modelo queda entonces como sigue (las variables con el signo - son exógenas):

(1) $T C R=T C R \frac{\left(Y^{T}\right)}{E}$

(2) $Y C S P N o C U=Y C S P N o C U(Y)$

(3) $\operatorname{Imp}=\operatorname{Imp}(Y, T C R)$

(4) $C s t r=\operatorname{Cstr}(Y P D, C s t r .1)$

(5) $Y=C s t r+\bar{I}+\bar{G}+\overline{T r}+\bar{X}-\operatorname{Imp}$

(6) $E=C s t r+\bar{I}+\bar{G}+\overline{T r}$

(7) $Y p d=Y-\overline{P F N R}-\left(\overline{Y C S P C} u+\overline{Y_{C S P N o C}}\right)-\overline{O Y S P}$

donde:

$\begin{array}{ll}T C R & : \text { Tipo de cambio real } \\ Y & : \text { PIB } \\ Y^{T} & : \text { PIB de tendencia } \\ E & : \text { Absorción interna } \\ Y c S P N o C u & : \text { Ingresos corrientes del sector público } \\ & \text { no vinculados al cobre } \\ \text { Imp. } & : \text { Importaciones } \\ \text { Cstr } & : \text { Consumo privado sin transferencias } \\ I & : \text { Inversión privada } \\ G & : \text { Consumo e inversión públicos } \\ T r & : \text { Transferencias del sector público al } \\ & \text { sector privado } \\ X & : \text { Exportaciones } \\ Y p d & : \text { Ingreso privado disponible } \\ P F N R & : \text { Ingreso neto de factores con el resto } \\ & \text { del mundo } \\ Y C S P C u & : \text { Ingresos corrientes del sector público } \\ & \text { vinculados al cobre } \\ \text { OYSP } & \text { : Otros ingresos del sector páblico }\end{array}$

${ }^{9}$ EI PIB de tendencia se calcula haciendo crecer el PIB del períado inmediatamente anterior al perfodo inicial, en este caso 1987, a la tasa de crecimiento media del período 1988-1993.
Las primeras cuatro ecuaciones son de comportamiento y las últimas tres son identidades básicas de contabilidad nacional. Para poder realizar simulaciones es necesario dar a las ecuaciones (1)-(4) una for" ma funcional específica, la que se presenta a continuación: ${ }^{10}$

(I) $T C R_{t}=T C R_{t-1} \cdot\left(\left(\left(Y_{t}^{T / Y_{t-1}^{T}}\right) \cdot\left(E_{t-1} / E_{t}\right)\right)^{\wedge a}\right) \cdot a_{1}$

(2) $Y_{C S P N o C} u_{j}=B_{0}+Y_{C S P N o C u} \cdot\left(\left(Y_{i-1}\right)^{\wedge} b I\right)$

(3) $I m p t=I m p_{t \cdot 1} \cdot\left(\left(Y_{i}^{T} / Y_{t \cdot i}^{T}\right)^{\wedge C} I\right)^{*}\left(\left(T C R / T C R_{t-1}\right)^{\wedge} c_{2}\right)$

(4) $C s t r_{t}=D_{0}+d_{1} \cdot\left(Y_{p d}\right)+d_{2} \cdot\left(\right.$ Cstr $\left._{t-2}\right)$

Los coeficientes de estas ecuaciones se presentan en el cuadro 1.

Los valores supuestos para las elasticidades representan cifras plausíbles para ellos. Como se explicó, fue necesario agregar a las ecuaciones presentadas los ajustes de constantes que se presentan en el cuadro 2 . Esto se hizo para poder reproducir en forma exacta el comportamiento de la economía. Estos ajustes de constante representan los shocks exógenos que han afectado a las distintas variables de comportamiento, y que no son representados por los efectos de las variaciones en las variables exógenas.

Este modelo no ha sido estimado empiricamente, aunque los valores de las elasticidades empleadas se basan en estimaciones realizadas previamente, ya que su objetivo no es estimar empíricamente valores para los parámetros que explican el comportamiento macroeconómico en Chile sino ilustrar de manera simplificada las relaciones que representan tal comportamien-

CUAORO I

Coefleientes de las ecuaclones

\begin{tabular}{cr}
\hline Coeficiente & \multicolumn{1}{c}{ Valor } \\
\hline$a_{0}$ & 0.47000 \\
$\mathrm{a}_{1}$ & 1.03465 \\
$\mathrm{~B}_{0}$ & -23.00000 \\
$\mathrm{~b}_{1}$ & 1.20000 \\
$\mathrm{c}_{1}$ & 1.20000 \\
$\mathrm{c}_{2}$ & -0.40000 \\
$\mathrm{D}_{0}$ & 101.79045 \\
$\mathrm{~d}_{1}$ & 0.53819 \\
$\mathrm{~d}_{2}$ & 0.49219 \\
\hline
\end{tabular}

${ }^{10}$ El subíndice $t$ en las variables representa el período. Las ecuaciones presentadas ignoran la variable de ajuste. 
CUADRO 2

Chile: Ajustes de consitantes

\begin{tabular}{|c|c|c|c|c|}
\hline & $\begin{array}{c}\text { Tipo de } \\
\text { cambio real }\end{array}$ & $\begin{array}{l}\text { Ingresos corriemtes } \\
\text { del sector público } \\
\text { (no cobre) }\end{array}$ & Impuestos & $\begin{array}{l}\text { Consumo privado, } \\
\text { sin transferencias }\end{array}$ \\
\hline 1981 & -8.60 & -4.69 & -13.00 & 282.66 \\
\hline 1982 & -5.22 & -147.61 & -217.34 & $-370,60$ \\
\hline 1983 & 5.02 & -82.11 & -66.79 & -215.31 \\
\hline 1984 & 1.27 & 48.14 & 53.13 & -9967 \\
\hline 1985 & 10.94 & -41.44 & -49.39 & $-385,99$ \\
\hline 1986 & 5.03 & -43.77 & 42,46 & -340.13 \\
\hline 1987 & 2.03 & -0.25 & 110.78 & -249.18 \\
\hline 1988 & 3.56 & -73.62 & 70.84 & $-150,30$ \\
\hline 1989 & -3.69 & -110.79 & 132.92 & -62.53 \\
\hline 1990 & -2.90 & $-80.2 B$ & -0.65 & -284.69 \\
\hline 1991 & -11.38 & 82,46 & -53.44 & $-277,05$ \\
\hline 1992 & -9.71 & -11.70 & 118.65 & -214.07 \\
\hline 1993 & -1.10 & -28.79 & 99.19 & -273.72 \\
\hline
\end{tabular}

to en el país. El modelo se utiliza en la simulación de los efectos macroeconómicos de cambios en la política fiscal, en lo que respecta a la variabilidad de la tasa de crecimiento del gasto público y a la flexibilidad de las tasas de impuestos indirectos.

El ejercicio que se desarrolla a continuación tiene por objeto estudiar el efecto de la política fiscal sobre el comportamiento cŕclico del PIB en el período 1988-1993. Ese comportamiento es medido por la desviación estándar de la tasa de crecimiento del PIB respecto de la de tendencia. El efecto cíclico de la política fiscal es aislado mediante simulaciones que cotejan la política físcal vigente durante el perfodo estudiado con un esquema de política fiscal contracíclica, que estabiliza el crecimiento de la absorción pública y flexibiliza las tasas tributarias. Este esquema de política fiscal consiste en eliminar los impulsos cíclicos de origen fiscal mediante la estabilización de la tasa de crecimiento de la absorción pública real, y compensar impulsos cíclicos de origen privado mediante cambios en la tasa de ciertos impuestos indirectos.

\section{Ejercicios de simulación}

Se realizaron tres simulaciones (cuadro 3). La simulación 1 se utilizó como base para calibrar el modelo con los datos efectivos. La simulación 2 consistió en modificar el gasto público, manteniendo la tasa de crecimiento de la absorción pública constante en todo el período; se realizó sin alterar la tasa de impuestos con el objeto de distinguir el impacto contracíclico atribuible al control del gasto público. Por último, la sinulación 3 incorporo, junto con la tasa constante de creci- miento de la absorción pública, aumentos o disminuciones transitorios de las tasas de ciertos impuestos indirectos con fines contracíclicos.

El rendimiento de los aumentos o disminuciones transitorios de tasas de impuestos fue limitado a un valor equivalente al $0.5 \%$ del pIB anual, dadas las dificultades prácticas para conseguir cambios mayores en la recaudación tributaria. Para obtener una recaudación adicional equivalente a medio punto del PIB anual se requiere un aumento de un punto en la tasa del impuesto al valor agregado (IVA) vigente por todo un período anual, por lo que este esquema requeriría una banda para la tasa del Iva de $+/$ - un punto porcentual, ${ }^{11}$ Todos los aumentos de recaudación fueron destinados a ahorro, ya que la tasa de crecimiento de la absorción pública se mantuvo inalterada.

\section{Resultados de la simulación}

La variabilidad de la tasa de crecimiento del PIB fue notable: en el periodo 1988-1992 fluctuó entre 3.0\%

\footnotetext{
" No se puede descartar a priori el uso de otros impuestos indirectos con estos propositos, pero dada su cobertura, facilidad de administración y rapidez de respuesta, el jva parece el instrumento más eficaz para este fin. Una razón para limilar la variación de la tasa del iva descansa en el argumento terrico de que es óptimo evitar las alteraciones a las tasas tributarias para evitar distorsiones en los precios relativos y que se genere incertidumbre respecto de las políticas. (Véase los fundamentos de este argumento en Barro, 1979, y una breve explicación de él en Blanchard y Fischer, 1989). El argumento microeconómico en favor de una tasa de impuestos constante es contrapesado al menos en parte por el argumento macroeconómico de la menor incertidumbre que resulta de una variación cíclica más moderada del PIB, a la que precisamente contribuye una tasa de impuestos ajustable.
} 
CUADRO 3

Ejercicios de simulación de política fiscal contracíclica, período 1988-1993 (Porcentajes)

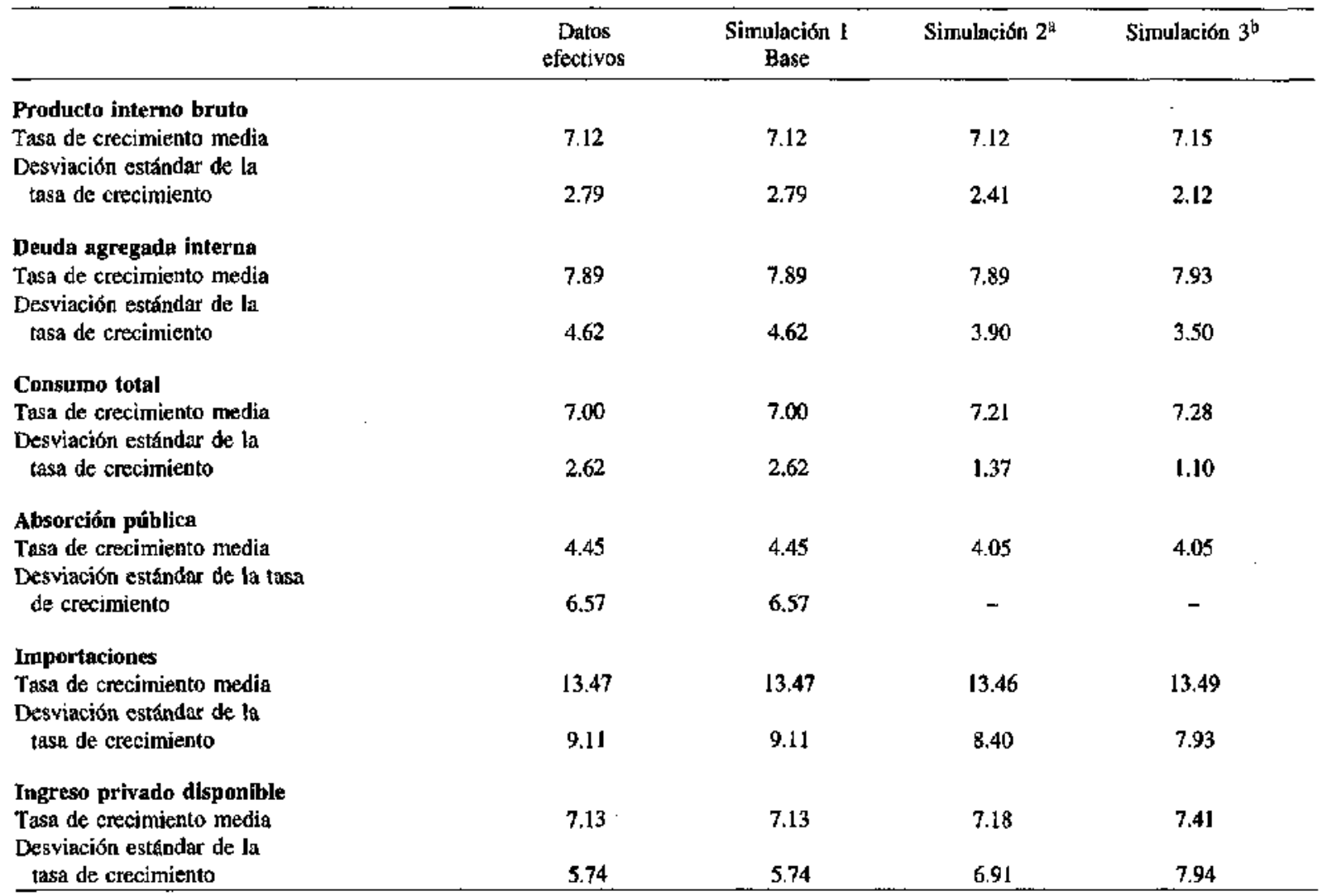

a Se modifić el gasto público, manteniendo constante la tasa de crecimiento de la absorción pública y la tasa de impuestos.

b Junto con la tasa constante de crecimiento de la absorción pública se incorporaron aumentos o disminuciones transitorios de ciertos impuestos indirectos.

y $11 \%$ (gráfico 2). Un simple intervalo para la tasa de crecimiento del pIB a un nivel de confianza del $95 \%$ la situaría entre $2.4 \%$ y $12.6 \%$, dada la desviación estándar observada en la muestra. Esto indica una importante incertidumbre respecto al comportamiento futuro de la actividad económica.

GRAFCO 2

Chile: Tesas de variaclón del producto interno bruto en 12 meses

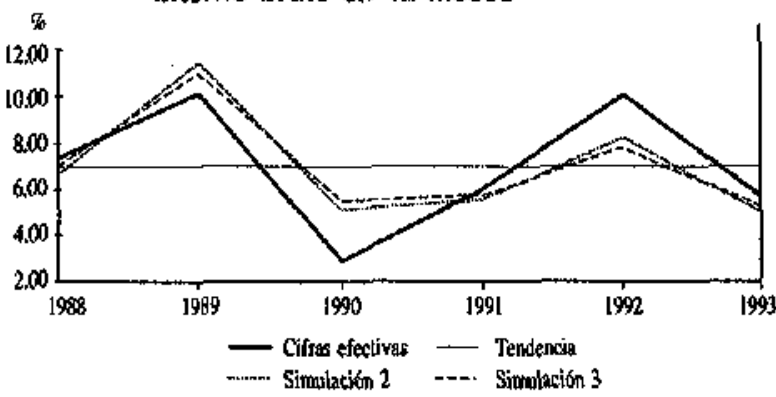

Según los ejercicios de simulación, la política fiscal contracíclica reduciría en $24 \%$ la variabilidad de la tasa de crecimiento del PIB, representada por su desviación estándar, lo que obedecería casi en partes iguales a la estabilización de la tasa de crecimiento de la absorción pública y a la variabilidad de la tasa tributaria. El crecimiento constante del gasto público disminuiría la variabilidad del PIB en $14 \%$, y el $10 \%$ restante sería atribuible a la variabilidad de la tasa tributaria.

Es notable la reducción que a lo largo del perío do ha tenido la relación entre la absorción pública y el producto. Desde 1988 a 1993 la tasa de crecimiento acumulativa del gasto público con significación macroeconómica ha sido inferior a la tasa de crecimiento del PIB (gráfico 3). En los ejercicios de simulación con política fiscal contraciclica la tasa de crecimiento de la absorción pública se mantuvo casi en los niveles de la simulación base, diferenciándose solo por el patrón de reducción más suave que aparece en la simulacio- 
GRAFICO 3

Chile: Absorción pública como \% del producto interno bruto

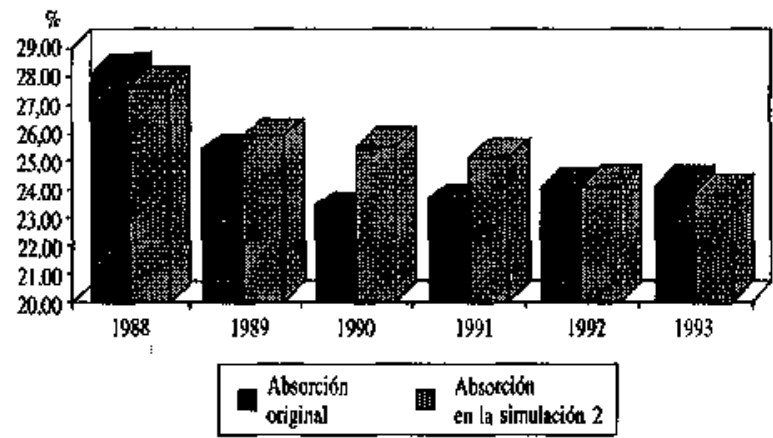

nes 2 y 3 como resultado de mantener constante la tasa de expansión del gasto. ${ }^{12}$

También es de significación la baja que ha experimentado en el período la participación en el PIB de los tributos no vinculados al cobre, los que no han alcanzado a recuperarse completamente después de la reforma tributaria de 1990. Cabe destacar asimismo que la variabilidad de la tasa de crecimiento de la tributación disminuyó en el escenario en que la tasa de impuestos es variable. Este resultado indica que el efecto de estabilización del crecimiento del PIB domino sobre el efecto de las variaciones contracíclicas en la tasa de tributación.

La variabilidad del consumo total disminuye al utilizarse una política fiscal contracíclica, pero la del consumo privado sin transferencias tiende a mantenerse. Esto se explicaría porque la variabilidad de la tasa de impuestos genera variabilidad en el consumo privado que no es compensada por la mayor estabilidad en el crecimiento del pIB. Efectivamente, la varianza del ingreso privado disponible aumenta cuando se aplica una política fiscal contracíclica como la descrita.

El balance fiscal global, incluido el déficit de caja estimado para el Banco Central, se hace más variable al aplicarse la política contracíclica. Teóricamente el resultado es correcto, ya que esto ocurre en la medida en que las acciones de política fiscal compensan los impulsos cíclicos de origen privado. $Y$ ese es el efecto que se acentúa cuando se utilizan los impuestos como instrumento para compensar impulsos cíclicos de origen no fiscal, ya que entonces la variabilidad del balance fiscal aumenta significativamente respecto del caso base, y se eleva solo marginalmente cuando se elimina el ciclo.

Los resultados obtenidos muestran que una parte no despreciable de la variabilidad del crecimiento del PIB puede atribuirse a la variabilidad del crecimiento del gasto público. Sin embargo, para eliminar el comportamiento cíclico del piB se requiere una política fiscal extraordinariamente flexible. Esta debería actuar de forma compensatoria a los impulsos ćclicos de origen privado, utilizando tributos contracíclicos, y evitar los impulsos cíclicos fiscales con el crecimiento de la inversión y el consumo públicos a tasa constante. En todo caso, queda claro que los impulsos cíclicos no pueden ser completamente contrarrestados por la política fiscal: de hecho, el $75 \%$ de la variabilidad cíclica del PIB no puede ser anulada por este medio.

\section{IV}

\section{Características y problemas de una propuesta de política fiscal anticíclica}

En este artículo se exploran las posibilidades que tiene el fisco de estabilizar el ciclo económico chileno, definido como la variabilidad de la tasa de crecimiento del PIB, usando los diferentes instrumentos que po-

\footnotetext{
${ }^{12}$ La tasa de aumento de la absorción pública se redujo marginalmente para evitar que en las simulaciones 2 y 3 aumentara ta tasa de crecimiento medio del PIB. La indivisibilidad del cambio en los tributos, que se mantuvo en aumentos o disminuciones de un punto del iva (cambios de $0.5 \%$ del prB), explica el aumento marginal de la tasa de crecimiento del PIB en la simulación 3 .
}

see. Hay bastante consenso con respecto a los efectos perjudiciales que tiene para la economía una variabilidad extrema de la tasa de crecimiento del PIB, como se ha dado en la economía chilena en períodos recientes. De hecho esa variabilidad tiene un efecto pernicioso sobre la inversión interna y por ende sobre las posibilidades de crecimiento futuras.

El hecho de que el fisco tenga algủn espacio para aminorar los vajvenes cíclicos aconseja estudiar algún mecanismo con este propósito, dada la importante variabilidad cíclica que ha presentado la actividad eco- 
nómica en Chile en los años noventa y los frecuentes shocks transitorios que ha sufrido en el pasado. Sin embargo, para avanzar en ese sentido la discusión presupuestaria tendría que darse en un plano de más largo plazo, quizás en términos de programas quinquenales de gasto. Esto es imposible en el marco político-legislativo actual, donde se discute respecto al nivel del gasto actual con referencia a los ingresos tributarios y no tributarios disponibles en la coyuntura.

Hay varios aspectos que deben ser examinados y precisados en relación con una propuesta de política fiscal contracíclica. Ellos son la naturaleza de las limitaciones que se establecerían al crecimiento dal gasto público, la índole y grado de flexibilidad de los impuestos y las características del fondo de estabilización fiscal.

\section{Restricciones al crecimiento del gasto público}

Una propuesta de política fiscal contracíclica consistiría en limitar el crecimiento del gasto público al crecimiento del PrB potencial. A fin de obviar el problema de medición del plB potencial, parecería práctico usar como estándar de referencia un promedio móvil de las tasas de crecimiento del PIB. Para evitar que las fluctuaciones cíclicas introduzcan demasiada variabilidad en la tasa de crecimiento del gasto público, parece conveniente que dicho promedio móvil se refiera a un período relativamente largo, por ejemplo diez años. No es descartable que en respuesta a objetivos estructurales del sector público la tasa de crecimiento de la absorción pública pueda ser menor o mayor que la tasa media de crecimiento del PIB en los tíltimos 10 años.

Dado que el objetivo de la propuesta es contener el impacto sobre la demanda agregada de bienes y servicios, el gasto público que se debería limitar es el correspondiente a la absorción pública. Esta incluye los gastos en consumo e inversión del gobierno central que generan presiones sobre la demanda agregada de bienes y servicios, incluyendo sueldos y salarios, compras de bienes y servicios y transferencias. Por lo tanto, en relación con los conceptos de gasto utilizados actualmente en la elaboración del presupuesto público, sería recomendable excluir para este efecto las partidas correspondientes a la inversión financiera (préstamos) y a los intereses pagados sobre la deuda pública interna (fundamentalmente al Banco Central) y externa.

Es indudable que cualquiera sea la forma en que se restrinja el crecimiento del gasto públiç, deben dejarse ciertos grados de flexibilidad y algunas válvulas de escape para modificar los límites en deterninadas circunstancias. En particular, cl gobiemo debe conservar cierta discrecionalidad en cuanto al nivel de los gastos de inversión pública, $o$ ante situaciones de emergencia. Los desvíos en estos casos deberían ser por motivos justificados y estar sujetos a compensación en los años siguientes al período para el cual se elaboró el presupuesto.

\section{Flexibilización de impuestos}

El principal candidato a la flexibilización es sin duda el IVA, cuya tasa ya fue parcialmente flexibilizada en el acuerdo tributario aprobado por el Congreso que permitió al gobierno fijarla entre $16 \%$ y $18 \%$ a partir de 1996. El uso del Iva como herramienta de estabilización cíclica tiene varias ventajas importantes. En primer lugar, su amplia cobertura hace que basten cambios menores en la tasa de tributación para lograr un efecto significativo en la recaudación. Las modificaciones razonables en la tasa del IVA son más efectivas y generan menos distorsiones que los cambios en otras tasas de tributación. Además, el IYA es el impuesto que afecta más directamente el gasto en consumo. Y, por último, la respuesta de la recaudación a un aumento de su tasa es relativamente inmediata y no genera mayores problemas de evasión y administración.

El impacto de una mayor tasa de IVA sobre el gasto se produce porque el impuesto adicional reduce el ingreso disponible, del cual el gasto en consumo depende en forma importante; esto hace que el manejo procíclico de esa tasa sea un elemento estabilizador. Por otra parte, como las modificaciones transitorias de la tasa del jva cambian el costo relativo de gastar en el presente, comparado con el de gastar en el futuro, las fluctuaciones procíclicas de esta tasa pueden influir mucho en la estabilización de la compra de bienes durables afectos al IVA.

Pese a su positivo efecto sobre el nivel de actividad, la flexibilización de la tasa del Iva tiene un costo que debe considerarse; su manejo procíclico tenderá a afectar la tasa de inflación medida en el corto plazo, lo que impone ciertos límites al uso potencial de este mecanismo. Como se sef̂aló en la sección anterior, para variar la recaudación tributaria en $1 \%$ del PIE es preciso modificar la tasa del IYA en alrededor de 2 puntos porcentuales. Asimismo, el costo señalado subraya la necesidad y conveniencia de avanzar en el proceso de reducir la importancia práctica de los mecanismos de indexación que aumentan la vulnerabilidad de la tasa de inflación a choques de costos.

Este límite al uso del IVA como herramienta estabi- 
lizadora sugiere estudiar la conveniencia de complementar este mecanismo con alguna flexibilización de los impuestos directos (a la renta especialmente), los que no tienen incidencia directa sobre los costos y los precios. Sin embargo, dado que la recaudación por impuestos a la renta es relativamente menor, serían necesarios cambios muy significativos en las tasas de tributación para elevar los recursos recaudados en forma medianamente relevante, lo que tendría efectos altamente distorsionadores. Además, debido a problemas de cobertura y evasión, en el corto plazo el aumento de la tasa de los impuestos a la renta tendería a afectar fundamentalmente a los asalariados. Por último, cabe considerar que, por la naturaleza de su diseño actual, los impuestos a la renta son ya bastante estabilizadores. ${ }^{13}$

Las opciones anteriores pueden complementarse con la flexibilización de los incentivos tributarios a la inversión. Estos incentivos significan un monto menor en términos de recaudación tributaria, pero pueden contribuir mucho a estabilizar más el gasto durante el ciclo, porque permiten alterar la rentabilidad relativa de invertir en el presente respecto a la de postergar la inversion. El riesgo de usar este instrumento es que puede abrir espacios para la evasion tributaria.

$\mathrm{Si}$ consideramos que las alzas de impuestos se producirían en escenarios de fuerte expansión de la demanda agregada donde existe algún grado de peligro inflacionario, y de cafdas en escenarios fundamentalmente recesivos, resultará que las expectativas que se anticipan a la política tributaria se convertirán en un elemento procíclico. Por lo tanto, el momento oportuno para reducir o alzar los impuestos será diferente según la manera en que se forman las expectativas y el grado de elasticidad de la demanda frente a ellas.

Ahora bien, supongamos un escenario con expectativas y agentes que realizan sustitución intertemporal y que por lo tanto perciben los precios relativos de consumir en el presente o en el futuro (típicamente respecto de bienes durables). Si la economía atraviesa por un período recesivo y se prevé una baja en los impuestos, en el período que transcurre entre el anuncio y la caída se produce una contracción de la deman$\mathrm{da}$; pero independientemente de que se haya o no anun-

\footnotetext{
${ }^{3}$ En el caso del impuesto a las utilidades, esto se debe a que la base del impuesto tiene una fluctuación cíclica bastante más acentuada que la del producto. En el caso de los impuestos a las personas, se debe a so progresividad: cuando los ingresos personales suben, se recauda más q̨ue proporcionalmente, $y$ lo contrario ocurre cuando los ingresos personales bajan.
}

ciado, a medida que se difunde en el mercado la idea de que se avecina una baja de la tasa se comienza a producir una contracción de la demanda y los agentes retrasan sus decisiones de inversión en bienes durables. Si la economía está ante una erupción inflacionaria, los agentes terminarán anticipando sus decisiones de consumo de bienes durables, produciendo nuevamente un efecto procíclico.

La eficacia de una medida de flexibilización de los tributos depende en gran parte de la capacidad del fisco de anticiparse a la formación de las expectativas de los agentes, y por ende, de la oportunidad con que se efectúen los ajustes de los impuestos.

\section{Fondo de estebilización: naturaleza y caracte- risticas}

El fondo de estabilización se concibe como un complemento a la política fiscal contracíclica y debe entenderse como la instancia para contabilizar y acumular las respuestas contracíclicas de la política fiscal. Definido así, dista mucho de representar un indicador global del resultado de la gestión fiscal. Tampoco pretende medir globalmente los resultados financieros estructurales ni aquella parte relacionada con el impacto directo del ciclo económico sobre las finanzas públicas. Estas características evitan la imposición de limitaciones drásticas sobre el manejo financiero del sector público. La creación de este mecanismo de estabilización, sin embargo, no resuelve el problema de que la restricción de solvencia sea debidamente internalizada por el sector público.

Por lo tanto, el fondo de estabilización que definimos a continuación debe verse ślo como un registro contable del ingreso y uso de los recursos adicionales derivados de la aplicación de la política fiscal contracíclica; se alimentaría de la recaudación adicional obtenida por cambios impositivos que se desvíen del nivel normal definido para el IVA, y de la rentabilidad — si la hay-que genere el fondo acumulado. Habría desacumulación en caso de realizarse giros por concepto de bajas en la recaudación debidas a tasas del IVA inferiores a las consideradas normales.

Con el fin de otorgar transitoriedad a este tipo de política, creemos necesario además imponer límites mínimo y máximo a la magnitud que puede alcanzar el fondo como porcentaje del PrB. Nuestra sugerencia es que el límite inferior sea cero y el superior $8 \%$ del PIB, lo que permitiría acomodar ciclos como los de la economía chilena. En el caso de alcanzarse alguno de estos límites, proponemos que se active un mecanis- 
mo automático de corrección de impuestos, mediante incrementos tributarios en el caso de haberse aicanzado el límite inferior, o por la realización de giros mediante la rebaja de impuestos en el caso de haberse alcanzado el límite superior. Recordemos que el gasto estaría restringido por la limitación global impuesta al crecimiento del gasto público.

Para no imponer una situación inmediata muy restrictiva en el manejo de este instrumento, creemos necesario definir como punto de partida un fondo de $2 \%$ del pir, lo que podría lograrse con el traspaso de parte de los recursos financieros depositados actualmente por el fisco en el Banco Central. Los recursos generados por este fondo deberán ser depositados en

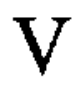

\section{Conclusiones}

Con el fin de evaluar cuantitativamente las distintas opciones que tiene el fisco chileno a su disposición, se han realizado varias simulaciones para el período 19881993, utilizando un modelo general, real y simple de tipo keynesiano. Este posee cuatro ecuaciones de comportamiento: consumo privado real, excluidas las transferencias públicas; tributos reales no vinculados al cobre; tipo de cambio real, e importaciones reales.

Los resultados obtenidos indican que el fisco puede conttibuir de manera importante a estabilizar el crecimiento del PIB. La desviación estándar del PIE se reduce $24 \%$ bajo una política fiscal contracíclica en ta cual la tasa de crecimiento de la absorción pública se iguala a la de crecimiento medio del piB, y se utiliza la tasa de los impuestos indirectos (VA) como elemento estabilizador.

En definitiva, parece razonable estudiar la posibilidad de planificar más las expansiones del gasto del fisco, de modo que ellas contribuyan a aminorar la amplitud del ciclo económico de la economía interna. El modelo muestra que al menos un $25 \%$ de la variabilidad del crecimiento puede ser reducida con una política de estabilización del crecimiento del gasto y con una política tributaria anticíclica. Sin embargo, es necesario examinar más cuidadosamente un conjunto de aspectos institucionales que se requerirían para garantizar la eficacia de esa política.

En primer lugar, respecto a la fijación de la tasa de crecimiento del gasto público, se debe analizar cuál el Banco Central y podrán ser administrados por éste para la recompra de su propia deuda. En cualquier caso, el instituto emisor se ocuparía de esterilizar las consecuencias monetarias de este mecanismo, como lo hace con cualquier otro tipo de depósitos del sector público.

Por último, en lo que toca a la tasa de interés que debiera pagar el Banco Central al fisco por los recursos en el fondo de estabilización, una primera alternativa sería la de una tasa igual a cero, que tendría la ventaja de aliviar el déficit de caja del instituto emisor. Otra alternativa, que parece más conveniente, sería la de otorgat a estos depósitos una tasa en dólares en condiciones iguales a las que entregan los pagarés del fisco con el Banco Central. es la medición más razonable del producto potencial, por un lado, y cuál es el período optimo que debiera considerar una planificación de los gastos públicos. En este trabajo hemos considerado que usar medias móviles de diez años y planificaciones quinquenales puede ser un criterio razonable y a la vez suficientemente de largo plazo; a este tema se vincula también el establecimiento de nuevas reglas para elaborar el presupuesto de la nación, sobre todo en lo que toca a grados de flexibilidad y sistemas de compensación intertemporal. Un aspecto institucional de crucial importancia es el establecimiento de un fondo de estabilización que sirva de colchón entre la variabilidad de la recaudación impositiva (que está sujeta a los ciclos económicos) y el gasto (que estaría sujeto a una regla fija). Dicho fondo debiera tener un límite inferior y uno superior, de modo que su ocupación sea transitoria y se modere el grado de discrecionalidad en su uso.

En segundo lugar, se debe estudiar la mezcla óptima de impuestos que se han de someter a un régimen de mayor flexibilidad, ya que el uso del IVA puede tener ciertos efectos desestabilizadores sobre la inflación de corto plazo. Como posibilidades para combinar con el IVA aparecen el impuesto a la renta y las franquicias tributarias a la inversión. Un aspecto importante que debe ser estudiado es la incidencia inequitativa en distintos sectores que se puede generar como producto del ajuste. También se debe conside- 
rar la potencial inexistencia de mercados de crédito para algunos sectores de la población, lo que puede dificultarles la capacidad de trasladar consumo intertemporalmente. Por otro lado, hay diferencias en la cobertura y los grados de evasión vinculados a los distintos impuestos, que pueden generar serias distorsiones a la hora de establecer una mayor flexibilidad. La eficacia de la política tributaria dependerá esencialmente de la capacioad de actuar de manera oportuna frente a cambios en las expectativas.

\section{Bibliografia}

Barro, R. J. (1979): On the determination of the public debt, Journat of Political Economy, vol. 87, № 5, parte 1, Chicago, llimois, The University Chicago Press,

Blanchard, O. y S. Fischer (1989): Lectures on Macroeconomics, Cambridge, Massachusedts, The MIT Press.

Blinder, A. y R. Solow (1989): Does fiscal policy maters?, A. Blinder (ed.), Macroecononics Under Debate, Ann Arbor, Michigan, University of Michigan Press.

Cortés, H. (1984): Lecciones del pasado: recesiones cconómicas en Chile: 1926-1982, Cuadernos de Economia, afio 21, No 63 , Santiago de Chile, Pontificia Universidad Católica de Chile, Instituto de Economía.

Christ C. F. (1967): A short-run aggregate-dernand model of the interdependence and effects of monetary and fiscal policies with the Keynesian and classical interest elasticities, The Anerican Economic Review, vol. LVII, No 2, Menasha, Wistonsin, American Economic Association.

Fleming J. M. (1962): Domestic financial policies under fixed and under floating exchange rates, Stuff Papers, vol. IX, $\mathrm{N}^{\circ} 3$, Washington, D.C., Fondo Monetario Internacional (FM).

Frenkel, J. A. y A. Razin (1987): Fiscal Policter and the World Economy, Cambridge, Massachusetts, The MIT Press.

Mundell R, A. (1963): Capital mobility and stabilization policy under fixed and flexible exchange rates, Canadian Journal of Economics and Political Science, vol. XXIX, $\mathrm{N}^{\circ} 4$, Toronto, Canadian Political Science Association.

Sachs J. D. y F. Larraín (1993): Macroeconomía en la econonía global, México, D.F., Prentice Hall Hispanoamericana, S.A. 